\title{
Betting on own knowledge: Experimental test of overconfidence
}

\author{
Blavatskyy, Pavlo R
}

\begin{abstract}
This paper presents a new incentive compatible method for measuring confidence in own knowledge. This method consists of two parts. First, an individual answers several general knowledge questions. Second, the individual chooses among three alternatives: (1) one question is selected at random and the individual receives a payoff if he or she has answered this question correctly; (2) the individual receives the same payoff with a probability equal to the percentage of correctly answered questions; (3) either the first or the second alternative is selected. The choice of the first (second) alternative reveals overconfidence (underconfidence). The individual is well calibrated if he or she chooses the third alternative. Experimental results show that subjects, on average, exhibit underconfidence about their own knowledge when the incentive compatible mechanism is used. Their confidence in own knowledge does not depend on their attitude towards risk/ambiguity.
\end{abstract}

DOI: https://doi.org/10.1007/s11166-008-9048-7

Posted at the Zurich Open Repository and Archive, University of Zurich

ZORA URL: https://doi.org/10.5167/uzh-25397

Journal Article

Published Version

Originally published at:

Blavatskyy, Pavlo R (2009). Betting on own knowledge: Experimental test of overconfidence. Journal of Risk and Uncertainty, 38(1):39-49.

DOI: https://doi.org/10.1007/s11166-008-9048-7 


\title{
Betting on own knowledge: Experimental test of overconfidence
}

\author{
Pavlo R. Blavatskyy
}

Published online: 13 August 2008

(C) Springer Science + Business Media, LLC 2008

\begin{abstract}
This paper presents a new incentive compatible method for measuring confidence in own knowledge. This method consists of two parts. First, an individual answers several general knowledge questions. Second, the individual chooses among three alternatives: (1) one question is selected at random and the individual receives a payoff if he or she has answered this question correctly; (2) the individual receives the same payoff with a probability equal to the percentage of correctly answered questions; (3) either the first or the second alternative is selected. The choice of the first (second) alternative reveals overconfidence (underconfidence). The individual is well calibrated if he or she chooses the third alternative. Experimental results show that subjects, on average, exhibit underconfidence about their own knowledge when the incentive compatible mechanism is used. Their confidence in own knowledge does not depend on their attitude towards risk/ ambiguity.
\end{abstract}

Keywords Overconfidence · Underconfidence $\cdot$ Lottery $\cdot$ Experiment $\cdot$ Risk aversion

JEL C91 · D 1

This paper investigates whether people think that they know more than they actually do. There is a substantial literature in psychology which suggests that people are apparently overconfident about own knowledge (e.g. Keren 1991; Yates 1990). In a typical experiment, subjects answer several binary-choice general knowledge questions. For each question, subjects have to choose which of the two suggested answers is correct in their opinion. Subjects are also asked to indicate their confidence on a $50-100 \%$ scale that their answer was correct. Individual responses are then sorted by the revealed confidence level and the percentage of correct

P. R. Blavatskyy $(\bowtie)$

Institute for Empirical Research in Economics, University of Zurich, Winterthurerstrasse 30,

CH-8006 Zurich, Switzerland

e-mail: pavlo.blavatskyy@iew.uzh.ch 
answers in each confidence category is calculated. Subjects are classified as overconfident if their stated confidence judgments are greater than the corresponding percentage of correctly answered questions.

Subjects are rarely overconfident in every confidence category. A typical finding is that subjects appear overconfident for difficult questions (percentage of correct answers below approximately $75 \%$ ) and underconfident or well calibrated for easy questions. This became known as the hard/easy effect (e.g. Lichtenstein and Fischhoff 1977). However, Juslin et al. (2000) recently conducted a meta-analysis of seventeen previous studies and found that the hard/easy effect is nearly eliminated when a researcher carefully controls for the scale-end effects (the upper and the lower bound on confidence scores) and linear dependency.

Gigerenzer et al. (1991) argue that subjects appear overconfident because an experimenter often selects non-representative general knowledge questions for which commonly used cues are not particularly useful. Gigerenzer et al. (1991) find that observed overconfidence is significantly reduced if a representative set of general knowledge questions is used in the experiment. This finding was replicated in several other studies (e.g. Juslin 1994; Winman 1997).

However, Griffin and Tversky (1992) and Suantak et al. (1996) argue that a representative set of general knowledge questions is often too easy for subjects and they reveal underconfidence due to the hard/easy effect. Juslin et al. (2000) conducted a meta-analysis of 95 studies with non-representative general knowledge questions and 35 studies with representative general knowledge questions. Juslin et al. (2000) found that the overconfidence phenomenon is less pronounced in the latter studies even after controlling for the difficulty level of both samples.

People often appear overconfident for questions where they possess a selfdeclared expertise (Heath and Tversky 1991) but their overconfidence decreases for questions where they find themselves incompetent (Kruger 1999). Erev et al. (1994) argue that different research methods can generate both an apparent over confidence and an apparent underconfidence from the same data, if we allow for the possibility that true judgments are perturbed by random errors. Soll (1996) points to a considerable heterogeneity in the individual data-some people appear to be systematically overconfident whereas others are biased to underconfidence.

Psychological studies of confidence in own knowledge typically do not provide financial incentives for revealing subjective confidence in an experiment. In contrast, economic literature on overconfidence usually employs monetary payoffs. Camerer and Lovallo (1999) find strong evidence of overconfidence in an experimental market entry game. Kirchler and Maciejovsky (2002) observe overconfidence according to subjective judgments but not according to revealed choices in an experimental asset market. Hoelzl and Rustichini (2005) find that overconfidence changes to underconfidence when an experimental task becomes less familiar and this effect is stronger with monetary incentives.

However, these economic papers do not measure confidence in own knowledge. They document an above average effect-people perceive themselves more favorably than they perceive the average member of their reference group. The above average effect does not necessarily indicate that people overestimate their group ranking in terms of general knowledge, ability, etc. Such apparent overconfidence may also reflect a subjective belief that the distribution of general 
knowledge, ability, etc. is highly skewed in the relevant reference group (related discussion is in Viscusi and Zeckhauser 2006; Krajc and Ortmann 2008).

When it comes to the measurement of confidence in own knowledge with monetary incentives, the most popular method is arguably an elicitation of confidence intervals (e.g. Russo and Schoemaker 1992). In a typical setting, subjects are asked to reveal a lower and upper bound for the $n$-percent confidence interval of a correct answer to a general knowledge question. Subjects are classified as overconfident if a correct answer falls into the stated interval in less than $n$-percent of cases. Using this method, Cesarini et al. (2006) observe that overconfidence is reduced by about $65 \%$ when monetary incentives and a frequency rather than a probability assessment are introduced. However, the confidence interval elicitation method is not incentive-compatible because subjects can deliberately misrepresent their confidence intervals (as explained in detail in the next section).

This paper presents a new incentive-compatible method for measuring individual confidence in own knowledge and/or ability. In the proposed method, overconfidence is tested by means of a simple (non-strategic) choice problem. Subjects receive an opportunity to bet either on their own knowledge and/or ability or on an equivalent lottery. Subjects who decide to bet on own knowledge and/or ability are classified as overconfident and subjects who decide to bet on the risky gamble- as underconfident. Unlike other methods of measuring individual confidence, such as the estimation of confidence intervals, our proposed method is incentive-compatible. Subjects cannot increase their monetary payoffs through deliberate misreporting of their confidence assessment, through conscious incorrect answering or through strategically chosen low effort.

The paper also presents the results of an experiment, where the proposed method is used for measuring individual confidence in general knowledge trivia. Experimental results show that subjects, on average, exhibit underconfidence about their own knowledge when the incentive compatible mechanism is used. Their subjective confidence does not depend on their risk/ambiguity aversion or seeking i.e. our proposed measure of confidence in own knowledge is not confounded with individual risk attitudes or attitudes towards ambiguity.

The remainder of this paper is organized as follows. Section 1 describes a new incentive-compatible method for measuring individual confidence in own knowledge. Section 2 presents the design and implementation of the experiment. Section 3 presents the experimental results. Section 4 concludes.

\section{Method}

As we already mentioned in the introduction, a popular method for measuring individual confidence is an elicitation of confidence intervals. In a typical setting, subjects are asked to reveal a lower and upper bound for the $n$-percent confidence interval of a correct answer to a general knowledge question, a future price in the experimental market, a ranking of their ability level etc. Subjects are classified as overconfident if a variable of interest falls into the stated interval in less than $n$ percent of cases. Experimental papers in psychology typically do not provide monetary incentives for reporting confidence intervals. 
Experimental papers in economics usually offer a small reward to subjects if a variable of interest falls into the revealed confidence interval with the stated frequency. For instance, Cesarini et al. (2006) ask subjects to provide a $90 \%$ confidence interval for numerical answers to ten general knowledge questions. Subjects are then asked to assess if the correct answers indeed fall into the stated intervals and they are awarded approximately US $\$ 6$ for each correct assessment.

Despite the popularity of the method, elicitation of confidence intervals is not incentive-compatible. If subjects are not informed about the exact mechanism how they earn money before they state their confidence intervals, there is no financial incentive for revealing subjective confidence intervals. ${ }^{1}$ If subjects are informed about their payoff function at the beginning of an experiment, they can increase their monetary payoff if they deliberately misrepresent their confidence intervals. For instance, Cesarini et al. (2006) report that one subject anticipated subsequent development of the experiment and strategically misreported his stated confidence intervals - for nine questions this student reported extremely wide confidence intervals and for one question he made a point estimate (so that nine out of ten of correct answers would fall into the stated $90 \%$ confidence intervals with relative certainty).

To avoid such problems associated with payoffs contingent on the realization of a variable of interest inside the stated confidence intervals, we propose a new method for measuring individual confidence. The proposed method consists of two parts. In the first part, a subject is asked to answer $N>1$ general knowledge questions or to contribute his or her effort in $N$ tasks, where success depends on individual ability. The subject is informed that the more questions he or she answers correctly (the more tasks he or she completes successfully), the higher are the chances of getting a fixed monetary payoff $M$.

In the second part, the subject faces a choice between the following alternatives:

1. One of $N$ questions (tasks) is selected at random and the subject receives a fixed monetary payoff $M$ if his or her answer to the selected question was correct (the selected task was completed successfully);

2. The subject plays a risky lottery that yields a fixed monetary payoff $M$ with probability $n / N$, where $n$ is calculated as the number of correctly answered questions (the number of tasks completed successfully) in the first part;

3. Either alternative 1 or alternative 2 is selected at random.

Alternatives 1-3 yield identical distributions of monetary outcomes. Thus, a wellcalibrated subject is indifferent between all three alternatives. However, an individual who is overconfident about own knowledge and/or ability would prefer alternative 1 and an individual who is underconfident would opt for alternative 2 . Thus, we have a simple measure of individual confidence in own knowledge through a revealed choice.

\footnotetext{
${ }^{1}$ In this case, subjects state confidence intervals without any knowledge whether too wide or too narrow intervals will increase or decrease their monetary payoffs. Since subjects do not know how their choice of confidence intervals affects future monetary payoffs, they do not have financial incentives for revealing true confidence intervals.
} 
Notice that the proposed method is incentive-compatible because subjects cannot increase their payoff if they deliberately provide incorrect answers to some of the general knowledge questions or if they strategically contribute a lower effort in some of the tasks. In such cases subjects still face a choice among identical probability distributions at the second part of the experiment (although with less favorable odds). As long as individual preferences satisfy first-order stochastic dominance, subjects cannot gain from giving deliberately incorrect answers (or strategically lower effort). Thus, our method avoids the incentive problems associated with the elicitation of confidence intervals.

However, one can argue that the proposed measure of individual confidence in own knowledge might be confounded with individual risk aversion and/or ambiguity aversion. At the second part of the experiment, individuals are confronted with a choice between an ambiguous lottery (alternative 1) and a risky lottery (alternative $2)$. Thus, risk and ambiguity attitudes may affect the observed choices. To investigate this possibility, we run a controlled laboratory experiment, where we measure individual confidence according to the proposed method as well as individual risk aversion and ambiguity aversion. We find that observed subjective confidence does not depend on risk/ambiguity aversion or seeking.

\section{Experiment}

The experiment is designed to test individual confidence in own knowledge without self-assessment relative to the reference group because an individual may have little or no information about abilities of other subjects. Individual confidence is measured in a simple decision problem. Initially, subjects receive ten general knowledge questions, each with five possible answers (e.g. Table 1). Subjects are informed that the more questions they answer correctly, the higher is their potential payoff. Having answered all questions, subjects face a choice among three alternatives:

1. One of ten questions is selected at random and the subject receives $50 \mathrm{CHF}$ (US $\$ \sim 39$ ) if he or she answered this question correctly and 1 CHF (US \$ 1.28) if his or her answer was incorrect;

2. One card is randomly drawn from a box with ten cards numbered from 1 to 10 and the subject receives $50 \mathrm{CHF}$ when the number on the drawn card is smaller

Table 1 Multiple-choice general knowledge questions used in the experiment (with correct answers in italic type)

\begin{tabular}{llllll}
\hline 1. Earth equator is around ... 000 km long & 40 & 24 & 36 & 52 & 14 \\
2. Solar system consists of... known planets. & 13 & 8 & 12 & 9 & 17 \\
3. First Tour de France took place in year ... & 1898 & 1915 & 1903 & 1814 & 1938 \\
4. Which triple of notes contains C major? & A-C-B & D-F-A & C-D-G & F-C-D & $C-E-G$ \\
5. Ludwig van Beethoven wrote... symphonies & 15 & 9 & 41 & 13 & 104 \\
6. Electric frequency in central Europe is ... Hz & 220 & 110 & 50 & 66 & 85 \\
7. Human cell consists of... chromosomes. & 32 & 58 & 46 & 38 & 23 \\
8. Human body has... sense organs. & 4 & 5 & 6 & 7 & 8 \\
9. Sum of angles in a triangle is ... degrees & 360 & 380 & 60 & 90 & 180 \\
10. "Lord of the Rings" is based on a book by... & Tolkien & Tolstoy & Trotzki & Thomas & Trevier \\
\hline
\end{tabular}


than or equal to $n$ ( $1 \mathrm{CHF}$ otherwise). $n$ is calculated as a number of questions that the subject answered correctly ${ }^{2}$;

3. Either alternative 1 or alternative 2 is selected (the subject presses button "Both alternatives are the same").

Alternatives 1-3 yield identical distribution of monetary outcomes but subjects are not aware of this fact. Subjects are classified as overconfident (i.e. overestimating own knowledge) if they select alternative 1, as underconfident (i.e. underestimating own knowledge) - if they select alternative 2, and well calibrated - if they select alternative 3. Notice that alternative 1 involves an ambiguous lottery (betting on an uncertain event) and alternative 2 involves a risky lottery (betting on an event with a known probability). Thus, ambiguity averse subjects may be inclined to choose alternative 2 and risk averse subjects may prefer alternative 1 . To control for risk attitudes we measured risk aversion and ambiguity aversion of every subject.

The Holt and Laury (2002) method is used for measuring individual risk aversion. A subject is faced with nine lottery pairs $\left\{S_{i}, R_{i}\right\}, i \in\{1, \ldots, 9\}$. A safer lottery $S_{i}$ yields $20 \mathrm{CHF}$ with probability $i / 10$ and $16 \mathrm{CHF}$ otherwise. A riskier lottery $R_{i}$ yields $40 \mathrm{CHF}$ with probability $i / 10$ and $1 \mathrm{CHF}$ otherwise. In each of nine lottery pairs a subject can choose either a safer lottery, or a riskier lottery, or can declare indifference (in this case lottery is selected at random). A risk neutral expected utility maximizer chooses lottery $S_{i}$ when $i \leq 4$ and lottery $R_{i}$-when $i \geq 5$. A risk seeking individual switches from $S_{i}$ to $R_{i}$ when $i \leq 3$ and a risk averse individual-when $i \geq 5$.

A similar method is used for measuring individual attitudes towards ambiguity. A subject is confronted with nine lottery pairs $\left\{U_{j}, A\right\}, j \in\{1, \ldots, 9\}$. An unambiguous lottery $U_{j}$ delivers $50 \mathrm{CHF}$ with probability $j / 10$ and $1 \mathrm{CHF}$ otherwise. An ambiguous lottery $A$ delivers either $50 \mathrm{CHF}$ or $1 \mathrm{CHF}$ with unknown probabilities. A subject is classified as ambiguity neutral if he or she chooses lottery $A$ when $j<5$ and lottery $U_{j}$-when $j>6$. A subject is classified as ambiguity averse if he or she switches from $A$ to $U_{j}$ when $j \leq 4$. A subject is classified as ambiguity seeking if the switch occurs when $j \geq 6$.

All 18 lottery pairs that are used for eliciting individual risk attitudes are presented to subjects in a random order. At the end of the experiment one of the problems is selected at random and subjects receive their payoffs according to their choices in the selected problem (a random lottery incentive scheme). The realization of all random events is performed by subjects themselves, who draw a standard bridge card from a box with a specified distribution of cards inside.

The experiment was programmed in z-Tree (e.g. Fischbacher 2007) and conducted in the IEW experimental lab in Zurich. Forty eight undergraduate students of the University of Zurich (24 male and 24 female) participated in three experimental sessions on December 16 and December 20, 2005. On average, the experiment lasted $45 \mathrm{~min}$ and average earnings, including a 5 CHF show-up fee, were $30.50 \mathrm{CHF}$ (median earnings were $23 \mathrm{CHF}$ ). A translation of the experimental instructions is given in the "Appendix".

\footnotetext{
${ }^{2}$ Although subjects see the number $n$, they are not informed that this is exactly the number of their correct answers.
} 


\section{Results}

Thirty-one out of forty-eight subjects (65\%) are classified as underconfident, 14 $(29 \%)$ - as overconfident and only three subjects $(6 \%)$ - as well calibrated. Figure 1 shows that individuals with poor general knowledge (one to five correct answers) and good general knowledge (eight to nine correct answers) appear to be predominantly underconfident. Among those subjects who gave one to five correct answers, the proportion of underconfident subjects is significantly higher than $50 \%$ ( $\chi^{2}$ test $8.33, p=0.004$ ). Only individuals with average general knowledge (who answered six to seven questions correctly) exhibit both under- and overconfidence in similar proportions.

Figure 2 shows how individual confidence is related to risk aversion/seeking. The horizontal axis allows to differentiate between underconfident, well calibrated and overconfident subjects. The vertical axis shows the number $i \in\{1, \ldots, 9\}$ of a lottery pair in which a subject switches from a safer lottery $S_{i}$ to a riskier lottery $R_{i}$ in the Holt and Laury (2002) procedure. Recall that subjects face decision problems in a random order and, therefore, they are not restricted to select a unique switching point. Only twenty six subjects (54\%) in our experiment have a unique switching point. For the remaining subjects, Fig. 2 shows a vertical line that connects the highest number $\underline{i} \in\{1, \ldots, 9\}$ such that a subject always chooses lottery $S_{i}$ when $i \leq \underline{i}$ and the lowest number $\bar{i} \in\{1, \ldots, 9\}$ such that the subject always chooses lottery $R_{i}$ when $i>\bar{i}$.

Figure 2 shows that the level of confidence is not correlated with risk attitudes. Among underconfident subjects, 52\% are risk averse, 6\% - risk neutral and 10\%risk seeking (the remaining subjects cannot be classified). Among overconfident subjects, $64 \%$ are risk averse, $7 \%$-risk neutral and 14\% - risk seeking. Overconfident subjects are not significantly more risk averse than underconfident subjects ( $p$ value for Fisher's exact probability test is 0.6192).

Figure 3 shows that individual confidence is also not correlated with ambiguity aversion or seeking. Thirty-five percent of underconfident subjects are ambiguity averse, 10\% - ambiguity neutral and 3\% - ambiguity seeking. Forty-three percent of

\section{Number of subjects}

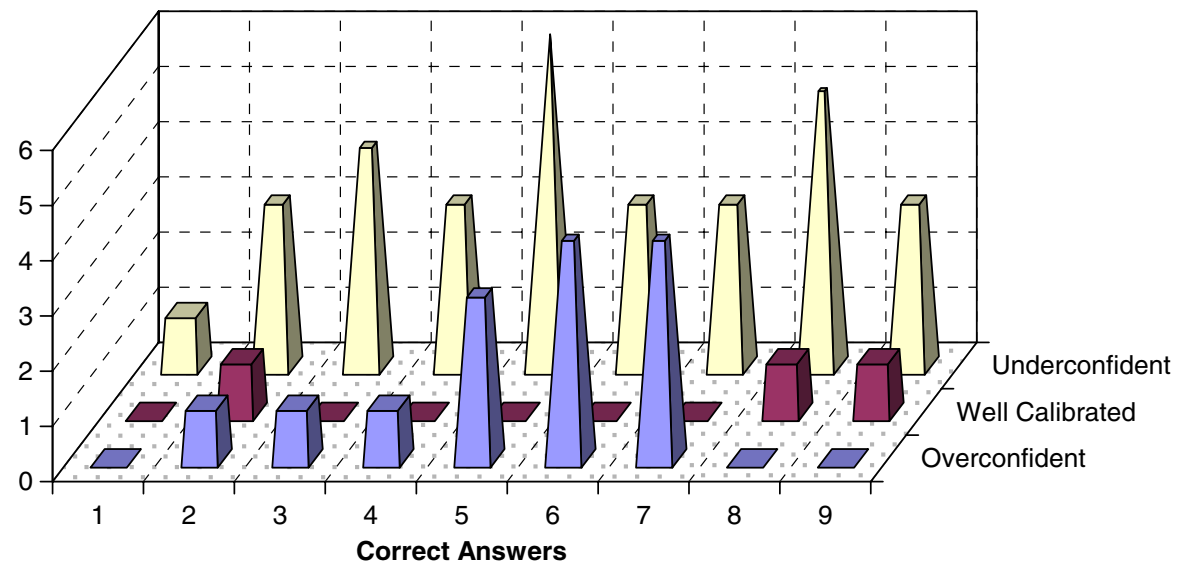

Fig. 1 Individual confidence and the number of correctly answered general knowledge questions 


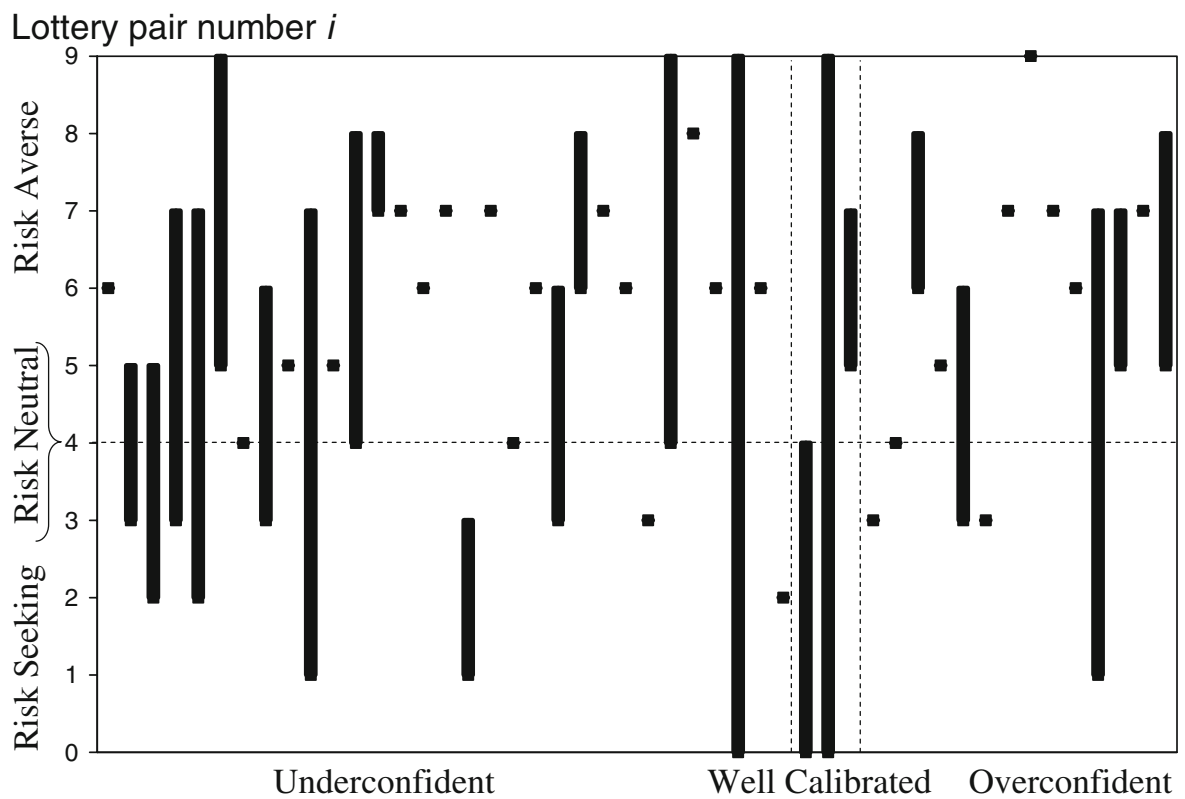

Fig. 2 Individual confidence and risk aversion/seeking

Lottery pair number $j$

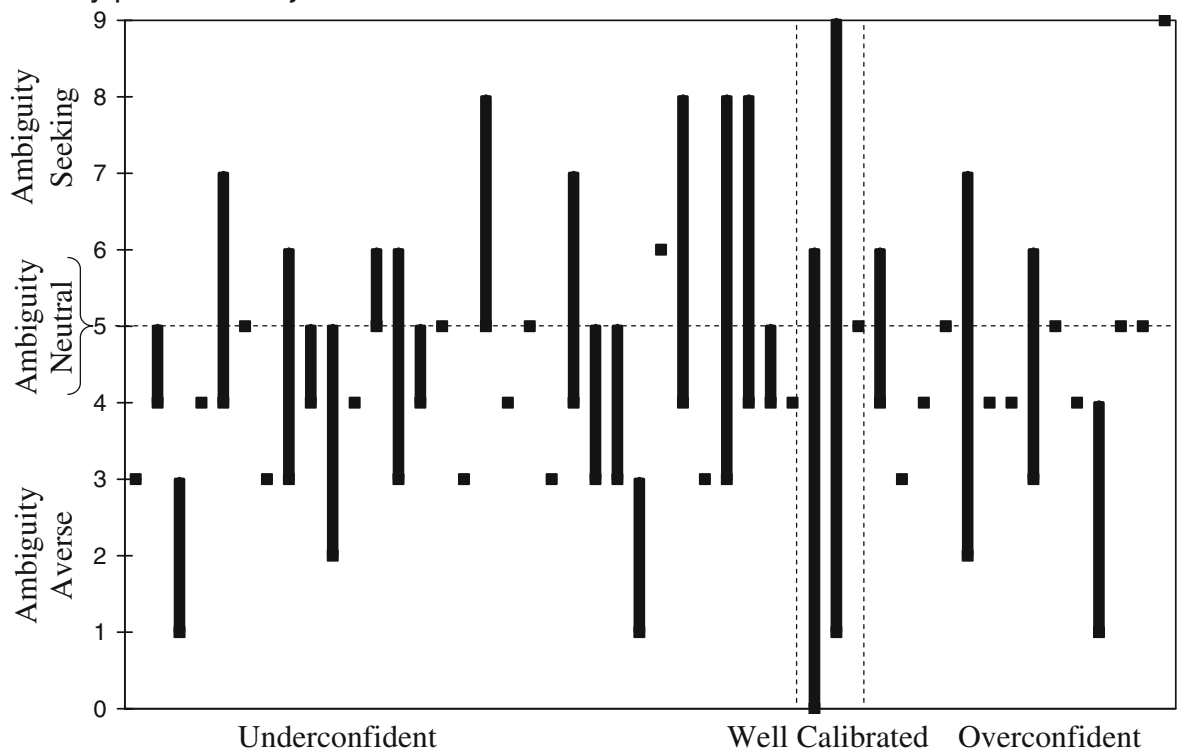

Fig. 3 Individual confidence and ambiguity aversion/seeking 
overconfident subjects are ambiguity averse, 29\%-ambiguity neutral and 7\%ambiguity seeking. Obviously, underconfident subjects do not appear to be more ambiguity averse than overconfident subjects. Thus, we can conclude that a high level of underconfidence observed in our experiment is not due to ambiguity aversion or risk seeking.

Experimental results clearly demonstrate that our proposed measure of individual confidence in own knowledge is not affected by individual attitudes towards risk or ambiguity. Experimental results also show that subjects appear to be predominantly underconfident about their own knowledge, when a new incentive compatible method is used. This is consistent with recent findings of Hoelzl and Rustichini (2005) and Cesarini et al. (2006) that monetary incentives tend to reduce the overconfidence bias.

\section{Conclusion}

Subjective confidence in own knowledge and/or ability is typically tested through the elicitation of confidence intervals. Unfortunately, this procedure is not incentivecompatible. This paper proposed a new incentive-compatible measure of individual confidence in own knowledge. Individual confidence is measured in a simple decision problem where subjects bet either on own knowledge/ability (which reveals overconfidence) or on an equivalent risky lottery (which signals underconfidence). The proposed measure is robust to possible strategic behavior of subjects, when they deliberately provide incorrect answers or contribute low effort. The results of a laboratory experiment show that the new measure of confidence in own knowledge is not confounded with individual attitudes towards risk or ambiguity.

Experimental results also show that individuals are predominantly underconfident. This conclusion does not depend on whether subjects have poor or good general knowledge, and whether they are risk/ambiguity averse or seeking. Our findings support the conclusion of Hoelzl and Rustichini (2005) and Cesarini et al. (2006) that monetary incentives tend to reduce (and even reverse) the overconfidence bias. Our results also suggest that this bias may be found in strategic games (e.g. Camerer and Lovallo 1999) because subjects receive little (or no) information about their counterparts, and not because they have excessive confidence in own ability.

Acknowledgement I would like to thank the editor and one anonymous referee for extensive comments on the previous version of this paper. I am also grateful to Ganna Pogrebna for programming the experiment in z-Tree and to Steffen Altmann, Franziska Heusi and Matthias Wibral for their assistance in running the experimental sessions.

\section{Appendix}

\section{Experimental instructions}

\section{Dear participant,}

You are about to participate in the experiment on individual decision making. The experiment will last about $1 \mathrm{~h}$. Your payoff will depend only on your decisions and 
the realization of random events (it will not be affected by the decisions of other participants in the room).

You will be given 19 problems and in each problem you need to choose "Left option", "Right option" or "Both alternatives are the same". If you choose "Both alternatives are the same", a computer will select either "Left option" or "Right option" at random. The problems will appear one by one on your computer screen. The example of a typical problem is given below:

Problem 5

\begin{tabular}{|c|c|c|c|}
\hline \multicolumn{2}{|l|}{$\begin{array}{l}\text { Box I contains: } \\
\qquad \begin{array}{l}\mathbf{5} \text { red cards } \\
\mathbf{5} \text { black cards }\end{array}\end{array}$} & \multicolumn{2}{|c|}{$\begin{array}{l}\text { Box J contains: } \\
\qquad \begin{array}{l}\mathbf{5} \text { blue cards } \\
\mathbf{5} \text { yellow cards }\end{array}\end{array}$} \\
\hline \multicolumn{3}{|c|}{$\begin{array}{l}\text { One card is drawn from the box. } \\
\text { You receive } \mathbf{2 0} \mathbf{C H F} \text { if this card is red } \\
\mathbf{1 6} \mathbf{C H F} \text { if this card is black }\end{array}$} & $\begin{array}{l}40 \mathrm{CHF} \text { if this card is blue } \\
1 \mathrm{CHF} \text { if this card is yellow }\end{array}$ \\
\hline Left option & \multicolumn{2}{|c|}{$\begin{array}{c}\text { Both alternatives are } \\
\text { the same }\end{array}$} & Right option \\
\hline Card is drawn from Box I & $\begin{array}{l}\text { Card is drav } \\
\text { Box I or }\end{array}$ & $\begin{array}{l}\text { either from } \\
\text { om Box } \mathbf{J}\end{array}$ & Card is drawn from Box \\
\hline
\end{tabular}

Your payoff is determined at the end of the experiment (when all participants answered all 19 questions). We will give one of the participants a box that contains cards numbered from 1 to 19 . The participant will draw one card from this box. The number on this card will determine one of 19 problems. This problem (together with your choice) will reappear on your computer screen. Then another contestant (it could be you!) will draw a card from the box THAT YOU HAVE CHOSEN in this problem. The color of this card will determine your payoff.

For example, suppose that the first drawn card has number 5 and problem 5 presented above reappears on your screen. And suppose that you have chosen "Right option" in this problem. Then one of the contestants will be asked to draw one card from Box J. If this card is blue, your payoff in the experiment is $40 \mathrm{CHF}$. If this card is yellow, your payoff in the experiment is $1 \mathrm{CHF}$.

Your payoff will be paid out in cash at the end of the experiment. All boxes used in the experiment are available for inspection after the experiment. If you have any further questions, please ask them now.

\section{References}

Camerer, C., \& Lovallo, D. (1999). Overconfidence and excess entry: An experimental approach. American Economic Review, 89(1), 306-318.

Cesarini, D., Sandewall, O., \& Johannesson, M. (2006). Confidence interval estimation tasks and the economics of overconfidence. Journal of Economic Behavior and Organization, 61, 453-470.

Erev, I., Wallsten, T., \& Budescu, D. (1994). Simultaneous over- and underconfidence: The role of error in judgment processes. Psychological Review, 101, 519-527.

Fischbacher, U. (2007). z-Tree: Zurich toolbox for ready-made economic experiments. Experimental Economics, 10(2), 171-178. 
Gigerenzer, G., Hoffrage, U., \& Kleinbölting, H. (1991). Probabilistic mental models: A Brunswikian theory of confidence. Psychological Review, 98, 506-528.

Griffin, D., \& Tversky, A. (1992). The weighing of evidence and the determinants of confidence. Cognitive Psychology, 24, 411-435.

Heath, C., \& Tversky, A. (1991). Preference and belief: Ambiguity and competence in choice under uncertainty. Journal of Risk and Uncertainty, 4(1), 5-28.

Hoelzl, E., \& Rustichini, A. (2005). Overconfident: Do you put your money on it? Economic Journal, 115 (503), 305-318.

Holt, C., \& Laury, S. (2002). Risk aversion and incentive effects. American Economic Review, 92(5), $1644-1655$.

Juslin, P. (1994). The overconfidence phenomenon as a consequence of informal experimenter-guided selection of almanac items. Organizational Behavior and Human Decision Processes, 57, 226-246.

Juslin, P., Winman, A., \& Olsson, H. (2000). Naïve empiricism and dogmatism in confidence research: A critical examination of the hard - easy effect. Psychological Review, 107, 384-396.

Keren, G. (1991). Calibration and probability judgments: Conceptual and methodological issues. Acta Psychologica, 77, 217-273.

Kirchler, E., \& Maciejovsky, B. (2002). Simultaneous over- and underconfidence: Evidence from experimental asset markets. Journal of Risk and Uncertainty, 25(1), 65-85.

Krajc, M., \& Ortmann, A. (2008). Are the unskilled really that unaware? An alternative explanation. Journal of Economic Psychology, forthcoming.

Kruger, J. (1999). Lake Wobegon be gone! The 'below - average effect' and the egocentric nature of comparative ability judgments. Journal of Personality and Social Psychology, 77(2), 221-232.

Lichtenstein, S., \& Fischhoff, B. (1977). Do those who know more also know more about how much they know? Organizational Behavior and Human Performance, 20, 159-183.

Russo, J. E., \& Schoemaker, P. J. (1992). Managing overconfidence. Sloan Management Review, 33, 7-17.

Soll, J. (1996). Determinants of overconfidence and miscalibration: The roles of random error and ecological structure. Organizational Behavior and Human Decision Processes, 65, 117-137.

Suantak, L., Bolger, F., \& Ferrell, W. (1996). The 'hard - easy effect' in subjective probability calibration. Organizational Behavior and Human Decision Processes, 67, 201-221.

Viscusi, W. Kip, \& Zeckhauser, R. J. (2006). National survey evidence on disasters and relief: Risk beliefs, self-interest, and compassion. Journal of Risk and Uncertainty, 33, 13-36.

Winman, A. (1997). The importance of item selection in 'knew-it-all-along' studies of general knowledge. Scandinavian Journal of Psychology, 38, 63-72.

Yates, J. F. (1990). Judgment and decision making. Englewood Cliffs, NJ: Prentice. 